\title{
SIMPLE TECHNIQUE FOR TWO-PHOTON ABSORPTION MEASUREMENTS IN POLYMERIC WAVEGUIDE CHANNELS
}

\author{
J.B.P. van Schoot, H.J.W.M. Hoekstra, A. Driessen and Th.J.A. Popma
}

MESA Research Institute, Lightwave Devices Group, University of Twente, PO-box 217, 7500 AE

Enschede,The Netherlands. X-31-53-894120 Fax:X-31-53-309547E-mail: sct@el.utwente.nl

\begin{abstract}
A simple technique for two-photon absorption measurements by pulse shape deformation in channel waveguides is described. Measurements on a ps time scale are performed. Results are in good agreement with literature values.
\end{abstract}

\section{Introduction}

The Two Photon Absorption (TPA) is an important parameter in determining whether a third-order nonlinear material is suitable for alloptical switching or not. ${ }^{1}$ We measured the TPA of the DANS-polymer of Akzo-Nobel by analysing the pulse shape deformation of pulses travelling through a $\mathrm{SiO}_{\mathrm{x}} \mathrm{N}_{\mathrm{y}}$ waveguide with a polymer cladding. In the presence of TPA the power in a channel waveguide can be written as: ${ }^{2}$

$$
P(z)=P_{0} \frac{\alpha}{\left(\alpha+\beta P_{0}\right) e^{\alpha z}-\beta P_{0}}
$$

where $\alpha$ and $\beta$ are modal linear absorption and two photon absorption respectively. $\mathrm{P}_{0}$ is the peak power at the on set $(\mathrm{z}=0)$ of the non-linear region. Instantaneous response is assumed. The modal two photon absorption coefficient is related to the material two photon absorption $\beta_{\mathrm{m}}$ according to:

$$
\beta=Q_{2} \beta_{m}
$$

where $\mathrm{Q}_{2}$ is an efficiency parameter which can be calculated from the variation theorem.2,3 Equation 1 is a nonlinear relation between the input and output power of a waveguide. Therefore pulses travelling through the waveguide will be deformed. The description of this deformation can be simplified to the one dimensional full width at half maximum value (FWHM). The model can be evaluated and pulse width changes can be determined for several values of $\alpha, P_{0} \beta$ and propagation length $L$. The relative FWHM is a function $f$ which can be written as:

$$
F W H M_{r e l}=f\left(\alpha_{D A N S}, P_{0} \beta\right)
$$

This function is shown graphically in figure 3 .

\section{Experimental set-up}

The waveguide is a 4 layer stack of $\mathrm{SiO}_{2}$ $(\mathrm{n}=1.45 @ \lambda=1.053 \mu \mathrm{m}), \mathrm{SiO}_{\mathrm{x}} \mathrm{N}_{\mathrm{y}}(\mathrm{n}=1.76$, $\mathrm{t}=0.2 \mu \mathrm{m}), \mathrm{Si}_{3} \mathrm{~N}_{4} \quad(\mathrm{n}=2, \mathrm{t}=0.1 \mu \mathrm{m})$ and $\mathrm{a}$ polymer layer serving as a cladding. In the coupling regions an index matching $\mathrm{SiO}_{\mathrm{x}} \mathrm{N}_{\mathrm{y}}$ layer is used instead (see figure 1). The ridge is defined with a $5 \mathrm{~nm}$ step in the $\mathrm{Si}_{3} \mathrm{~N}_{4}$ layer and has a width of $8 \mu \mathrm{m}$.

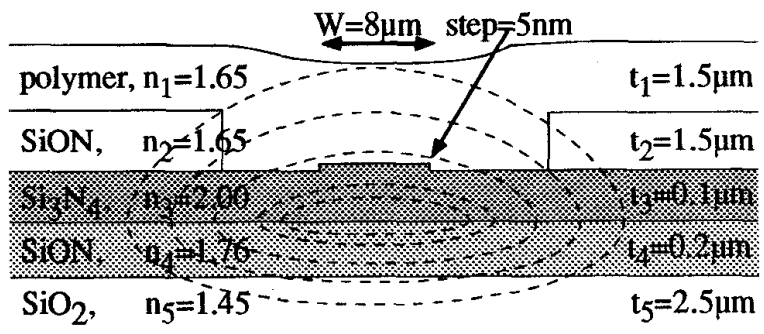

silicon

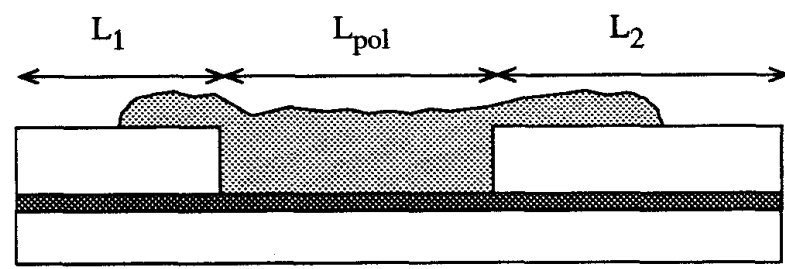

figure 1 The used channel structure. The intensity profiles give an impression of the localisation of the electrical field (upper). Cross section of the channel waveguide for the TPA measurements with relevant definitions. The optical power flows from left to right (lower).

The laser source is a mode-locked Q-switched $\mathrm{Nd}$ : YLF laser producing at a repetition rate of $134 \mathrm{~Hz}$ bursts consisting of 100 ps FWHM pulses. A pulse picker is used for picking the highest intensity pulse out of the pulse train. The trigger timing is shown in the inset of figure 2 . In this way high intensity pulses are obtained with a very low average power present 
in the suppressed which is important in avoiding thermal degradation of the end faces of the waveguides. We observed $75 \%$ of the optical power present in the high intensity pulses. The sampling oscilloscope is triggered on the high intensity pulses which are delayed with a mono modal fibre for compensating the internal delay of the oscilloscope. The observed signal shows a time jitter having a Gaussian time distribution with an RMS value of $50 \mathrm{ps}$. The pulse shape deformation measurements are performed on two channel waveguides with a length of $5 \mathrm{~cm}$. The length of the non-linear region is 1 and $4 \mathrm{~cm}$ respectively. The definitions are shown in figure 1.

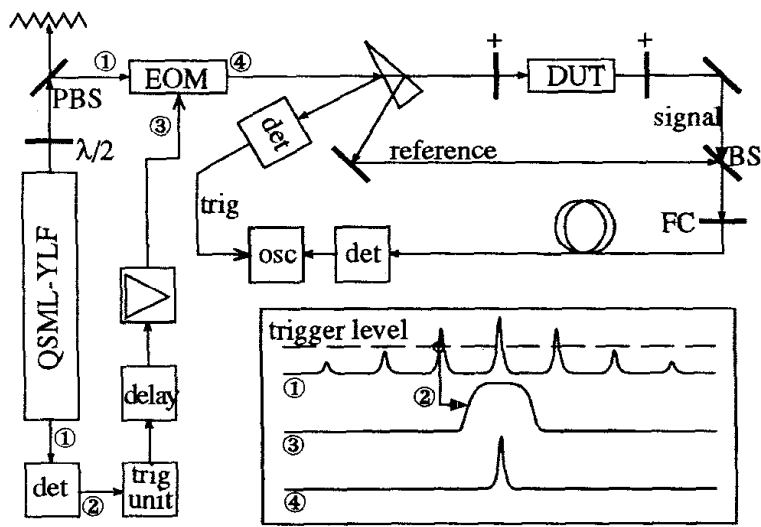

figure 2 The measurement set-up for the TPA measurements. The inset shows the timing at different positions in the set-up. Optical signals are represented by filled arrows. (P)BS: (polarising) beam splitter, EOM: electro-optic modulator, det: detector, OSC: sampling oscilloscope, $\lambda / 2$ : half wave plate, DUT: device under test.

\section{Results}

For the channels the input and output pulses are measured and averaged 32 times. The measured jitter is used to calculate the FWHM of the pulses from the measured FWHM.

\begin{tabular}{|c|c|c|c|c|c|c|}
\hline Device & \multicolumn{2}{|c|}{ channel 31} & \multicolumn{2}{|c|}{ channel 31} & \multicolumn{2}{|c|}{ channel 34} \\
\hline Quantity & in & out & in & out & in & out \\
\hline $\mathrm{L}_{1}[\mathrm{~mm}]$ & \multicolumn{2}{|c|}{10} & \multicolumn{2}{|c|}{10} & \multicolumn{2}{|c|}{5} \\
\hline $\mathrm{L}_{2}[\mathrm{~mm}]$ & \multicolumn{2}{|c|}{30} & \multicolumn{2}{|c|}{30} & \multicolumn{2}{|c|}{5} \\
\hline $\mathrm{L}_{\mathrm{pol}}[\mathrm{mm}]$ & \multicolumn{2}{|c|}{1} & \multicolumn{2}{|c|}{1} & \multicolumn{2}{|c|}{4} \\
\hline $\mathrm{W}[\mu \mathrm{m}]$ & \multicolumn{2}{|c|}{8} & \multicolumn{2}{|c|}{8} & \multicolumn{2}{|c|}{8} \\
\hline $\mathrm{P}[\mu \mathrm{W}]$ & 29 & 0.32 & 280 & 0.87 & 105 & 0.24 \\
\hline$P_{\text {peak }}[W]$ & 1100 & 12.5 & 10600 & 31 & 4180 & 8.6 \\
\hline $\mathrm{FWHM}_{\mathrm{rel}}$ & \multicolumn{2}{|c|}{0.992} & \multicolumn{2}{|c|}{1.062} & \multicolumn{2}{|c|}{1.118} \\
\hline
\end{tabular}

table 1 Experimental data used for the calculation of the TPA of the DANS polymer of Akzo Nobel.
The rise time of the detector and the sampling head are $7 \mathrm{ps}$ and $10 \mathrm{ps}$ respectively. The measured data are presented in table 1 and drawn as horizontal lines in figure 3.

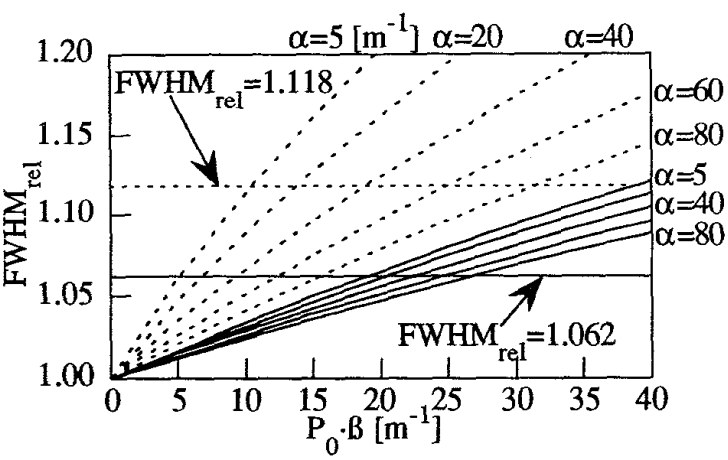

figure 3 The relation between the pulse width change and $P_{0} \cdot \beta$ for two different lengths of the non-linear interaction region. The horizontal lines correspond to the measurements.

$\cdots \cdot \cdot \mathrm{L}_{\mathrm{pol}}=4 \mathrm{~cm},---: \mathrm{L}_{\mathrm{pol}}=1 \mathrm{~cm}$.

With the aid of equations 1,2 and 3 the TPA of DANS can be calculated from the experimental data. The final conclusions to be drawn are:

$$
\begin{array}{ll}
- & \alpha_{\text {DANS }}=60 \pm 20\left[\mathrm{~m}^{-1}\right](2.5 \pm 1[\mathrm{~dB} / \mathrm{cm}]) \\
- & \alpha_{\text {SiON }}=25 \pm 15\left[\mathrm{~m}^{-1}\right](1 \pm 0.5[\mathrm{~dB} / \mathrm{cm}]) \\
\text { - } & \beta_{\mathrm{m}, \mathrm{DANS}}=4 \pm 2[\mathrm{pm} / \mathrm{W}]
\end{array}
$$

\section{Conclusions}

A simple method for determining the TPA is described. The value of the two photon absorption as given by Stegeman ${ }^{4}$ is $10 \mathrm{pm} / \mathrm{W}$ and is in reasonable agreement with our results.

\section{References}

1 Mizrahi, V., K.W. DeLong, G.I. Stegeman, M.A. Saifi and M.J. Andrejco, "Two-photon absorption as a limitation to all-optical switching", Opt. Lett., 14, 1140-1142, 1989.

2 Kogelnik, H., "Integrated Optics", in Guided-wave optoelectronics, Tamir, T., Ed., Springer Series in Electronics and Photonics. Springer-Verlag Publishing Company, 1975, ch. 2, 7-88.

3 van Schoot, J.B.P., "Characterisation and application of organic materials for all-optical integrated optic devices", Ph.D. thesis, University of Twente, Enschede, The Netherlands, 1994.

4 Stegeman, G.I. and A. Miller, "Physics of all-optical swtiching devices", in Photonics in switching, Midwinter, J.E., Ed. , vol. 1. Academic Press, Inc., 1993, chap. 5, pp. 81-145. 